\title{
EXTRAÇÃO, SECAGEM POR ATOMIZAÇ̃̃O E MICROENCAPSULAMENTO DE ANTOCIANINAS DO BAGAÇO DA UVA "ISABEL"(Vitis labrusca)
}

\author{
Extraction, spray drying and microencapsulating of 'Isabel' grape (Vitis labrusca) \\ bagasse anthocyanin
}

\author{
Eunice Valduga ${ }^{1}$, Leandra Lima² ${ }^{2}$ Roberta do Prado ${ }^{2}$,Francine Ferreira Padilha ${ }^{3}$, Helen Treichel ${ }^{4}$
}

\begin{abstract}
RESUMO
Objetivou-se obter neste trabalho um corante natural (antocianina), na forma de pó a partir do bagaço de uva da cultivar "Isabel" (Vitis labrusca), onde foram realizados estudos de extração e encapsulamento. Empregou-se o método de extração por imersão mediante técnica de planejamento experimental, onde as variáveis avaliadas foram $\mathrm{pH}$ da solução de extração $(1-2)$, volume de etanol $(100-250 \mathrm{~mL})$, tempo de extração $(3-7 \mathrm{~h})$ e temperatura de extração $\left(15-35^{\circ} \mathrm{C}\right)$. A concentração máxima de antocianinas totais obtidas foi de $300 \mathrm{mg} / 100 \mathrm{~g}$ de bagaço de uva (umidade $5 \%$ ) nos níveis inferiores de $\mathrm{pH}(1,0)$ e tempo (3 horas) e superiores de temperatura $\left(35^{\circ} \mathrm{C}\right)$ e volume de etanol $(250 \mathrm{~mL})$. Os extratos foram secos por atomização. A melhor condição para o encapsulamento e a secagem foi quando utilizaram-se proporções iguais de maltodextrina e goma arábica.
\end{abstract}

Termos para indexação: Antocianinas, Vitis labrusca, microencapsulamento, extração, bagaço de uva.

\begin{abstract}
This work had the aim of obtaining a natural pigment (anthocyanin) at powder form from 'Isabel' grape bagasse (Vitis labrusca), studying the extraction and encapsulating steps one applied the method of extraction by immersion by experimental design technique, when the factors investigated were $\mathrm{pH}$ of the extraction solution (1-2), volume of ethanol (100-250 mL), extraction time $(3-7 \mathrm{~h})$ and extraction temperature $\left(15-35^{\circ} \mathrm{C}\right)$. The maximum concentration of total anthocyanin was $300 \mathrm{mg} / 100 \mathrm{~g}$ of grape bagasse $\left(5 \%\right.$ moisture) at the lower levels of $\mathrm{pH}(1.0)$ and time $(3 \mathrm{~h})$, and at upper levels of temperature $\left(35^{\circ} \mathrm{C}\right)$ and amount of ethanol $(250$ $\mathrm{mL}$ ). The extracts were dried by a spray process. The best condition for encapsulating and drying was obtained when equal amounts of maltodextrin and Arabic gum were used.
\end{abstract}

Index terms: Anthocyanin, Vitis labrusca, microencapsulation, extraction, grape bagasse.

(Recebido em 27 de outubro de 2006 e aprovado em 2 de maio de 2007)

\section{INTRODUÇÃo}

As indústrias que processam a uva no Brasil são na sua maioria vinícolas que consideram o bagaço (cascas e sementes) de uva como subproduto. Esse subproduto tem recebido grande atenção por causa da grande produção na região sul do Brasil e por serem ricos em antocianinas.

Apesar de largamente disseminadas na natureza são poucas as fontes comercialmente utilizáveis de antocianinas. Entre essas fontes podem-se citar o resíduo da fabricação do vinho e do suco de uva que produz em o pigmento usado em alimentos, com o nome de enocianina (BOBBIO \& BOBBIO, 2001). Em alimentos industrializados, as antocianinas são empregadas como corantes naturais. Entretanto, sua utilização ainda é restrita pela baixa estabilidade em meios aquosos e pH acima de 2, condições bastante comuns durante o processamento e estocagem dos alimentos (FALCÃO et al., 2003).

As antocianinas são moléculas polares, em função dos grupos substituintes polares (hidroxilas, carboxilas e metoxilas) e glicosilas residuais ligados aos seus anéis aromáticos. Conseqüentemente, elas são mais solúveis em água do que em solventes não-polares, porém, dependendo das condições do meio, as antocianinas podem ser solúveis em éter. Essas características ajudam na extração e separação das antocianinas (HARBORNE, 1988).

Métodos convencionais de extração de pigmentos, usualmente empregam ácido hidroclorídrico diluído em metanol. $\mathrm{O}$ metanol contendo $0,001 \% \mathrm{HCl}$ foi mais efetivo, porém o $\mathrm{HCl}$ é corrosivo e o metanol é tóxico para o ser humano; conseqüentemente, os pesquisadores, que

1Doutora em Engenharia Química - Departamento de Engenharia de Alimentos - Universidade Regional Integrada do Alto Uruguai e Missões/URI Avenida 7 de Setembro, 1621, Campus de Erechim - Centro - 99700-000 - Erechim, RS - veunice@uricer.edu.br

${ }^{2}$ Graduadas em Engenharia de Alimentos - Departamento de Engenharia de Alimentos - Universidade Regional Integrada do Alto Uruguai e Missões/URI Avenida 7 de Setembro, 1621, Campus de Erechim - Centro - 99700-000 - Erechim, RS - leandralima@yahoo.com.br; roberta@yahoo.com.br

${ }^{3}$ Doutora em Ciência de Alimentos - Instituto de Tecnologia e Pesquisa(ITP) - Laboratório de Produtos Naturais e Sintéticos - Av. Murilo Dantas, 300 Prédio do ITP - Bairro Farolândia - 49032-490 - Aracaju, SE - francinepadilha@yahoo.com.br

${ }^{4}$ Doutora em Engenharia de Alimentos - Departamento de Engenharia de Alimentos - Universidade Regional Integrada do Alto Uruguai e Missões/URI -

Avenida 7 de Setembro, 1621, Campus de Erechim - Centro - 99700-000 - Erechim, RS - helen@uricer.edu.br 
trabalham com alimentos, preferem outros sistemas de extração (LOPES et al., 2000).

O processo de extração de antocianinas de uvas em leito fixo, adotando metanol como solvente foi estudado por Mantell et al. (2002). Xavier (2004) estudou os efeitos da temperatura de extração $(40,50$ e 60 o C) e do fluxo de recirculação (12, 17 e $22 \mathrm{mmol} / \mathrm{min})$, sobre o rendimento do processo. O único efeito significativo detectado foi a interação temperatura-fluxo de recirculação e foi atribuído ao forte controle da etapa de transferência de massa no processo operando a baixas temperaturas. Os valores da difusividade interna, no sólido, variaram de 3,53 a 5,02 (Dm $\mathrm{x} 1010 \mathrm{~m}^{2} / \mathrm{s}$ ).

Gao \& Mazza (1996) estudaram um processo de extração aquosa para antocianinas de girassol, demonstrando que a extração com água sulfurada (1000 ppm $\mathrm{SO}_{2}$ ) foi melhor do que a extração tradicional com etanol : ácido acético :água. Também foi demonstrado que 1 hora de extração foi suficiente para alcançar a extração completa dos pigmentos

A necessidade de conservação dos pigmentos tem incentivado o desenvolvimento de novas pesquisas neste setor, sendo que as formas mais importantes de conservação do pigmento são a encapsulamento e adição de antioxidantes. Entre os diferentes métodos, o secador por atomização (Spray dryer), é o método de secagem mais comum usado na indústria alimentícia por ser econômico, flexível e contínuo (DEL-VALLE, 2004).

Pouco ou quase nada foi encontrado na literatura aberta a respeito de encapsulamento de corantes, particularmente com respeito às antocianinas. Alguns artigos se referem a uma melhora da estabilidade de pigmentos (COUTINO, 2002; HUMEAUT et al., 2000; MALACRIDA \& MOTTA, 2006; WAGNER \& WARTHESEN, 1995). O‘Boyle et al. (1992) mostraram que â-ciclodextrina sozinha ou combinada com amido modificado, goma arábica e maltodextrina, é eficaz na microencapsulação de Dinitrosil Ferrohemochromo (DNFH), aumentando a vida de prateleira e a estabilidade à luz. No entanto, Caramez (1999) verificou que, ao encapsular antocianinas de Hibiscus sabdriffa L. com b-ciclodextrina, a degradação foi acelerada em níveis consideráveis. Wagner \& Warthesen (1995) encapsularam por secagem em Spray dryer, carotenos (suco de cenoura) com amidos hidrolisados (DE's 4, 15, 25 e 36,5) e constaram que o amido de maior DE proporcionou uma maior retenção de a e bcaroteno. Barbosa et al. (2005) encapsularam o pigmento bixina com goma arábica e maltodextrina e secaram em Spray dryer e observaram que a goma arábica proporcionou uma maior retenção do pigmento.
Nesse contexto objetivou-se, no trabalho, obter um corante natural (antocianina), na forma de pó, a partir do bagaço de uva da cultivar "Isabel" (Vitis labrusca), empregando-se a metodologia de planejamento de experimentos. Foram estudadas a extração, secagem por atomização e encapsulamento.

\section{MATERIAL E MÉTODOS}

\section{Coleta e Preparo de amostra}

O bagaço de uva da cultivar "Isabel" - Vitis labrusca foi seco a $40^{\circ} \mathrm{C}$, em estufa com ar circulante (Fanem SE-320), por 33 horas, atingindo umidade final de $5 \%$. Posteriormente, o bagaço foi triturado em multiprocessador (WALITA) e armazenado em frascos de vidro envoltos em papel alumínio, protegidos da luz e à temperatura ambiente.

\section{Extração de antocianinas}

Para definir as condições de extração das antocianinas pelo método de imersão em solvente, empregou-se a técnica de planejamento experimental do tipo Taguchi, no qual as variáveis estudadas foram: volume de etanol (100-250 mL), temperatura de extração $\left(15-35^{\circ} \mathrm{C}\right)$, pH (1-2) e tempo de extração (3-7 h).

Em cada experimento, foram utilizados 30 gramas de bagaço seco triturado e volume de solvente préestabelecido pelo planejamento experimental, em Erlenmeyer de $500 \mathrm{~mL}$. O recipiente foi envolto em papel alumínio, mantido em banho-maria com agitação a 600 rpm (Fisatom - modelo 752).

\section{Quantificação das antocianinas totais}

As antocianinas totais foram determinadas em espectrômetro (Agillent modelo 8453), no comprimento de onda $520 \mathrm{~nm}$, levando-se em conta o fator de diluição e o coeficiente de extinção molar da cianidina $(98,2)$ segundo método de Lees \& Francis (NIKETIC-ALEKSIC \& HRAZDINA, 1972). O resultado foi determinado conforme equação (1).

$$
A T=\frac{\Delta \cdot(\text { fator de diluição })}{E_{1 \mathrm{~cm} \cdot \lambda \operatorname{máx} x}^{1 \%}}
$$

Onde: AT: Antocianinas totais (mg/100 g); D: leitura de absorbância no espectrofotômetro;

Fator de diluição $=\frac{1^{a} \text { diluição } \cdot 2^{a} \text { diluição }}{\text { Quantidade retirada } 1^{a} \text { diluição }} ; \mathrm{E}_{1 \mathrm{~cm}}^{1 \%} \lambda_{\text {máx }}$ :

Absorbância de uma solução a 1\% do pigmento em Etanol$\mathrm{HCl}$, medida em cubeta de $1 \mathrm{~cm}$ no comprimento de onda de absorbância máxima. Esse valor corresponde a 990 para 
solução etanólica, segundo a recomendação de Guimarães et al. (1984).

\section{Encapsulamento das antocianinas}

O extrato foi concentrado em evaporador rotativo (Tecnal TE-210), a $80^{\circ} \mathrm{C}$ e pressão de $30 \mathrm{mmHg}$, por 2 horas. $\mathrm{O}$ volume final de solução foi $100 \mathrm{~mL}$ e pH de 3,5 (ajustado com tampão fosfato - $\mathrm{pH} 7,2$ ). Os agentes encapsulantes utilizados foram a maltodextrina (Mor-Rex 1920 DE 18- Corn Products Brasil) e a goma arábica (Vetec), que foram avaliadas através de um planejamento de experimentos completo $2^{2}$. As variáveis estudadas foram: Relação Maltodextrina:Goma (0:100, 50:50, 100:0) e Extrato (30-70 $\mathrm{mL}$ ). A homogeneização do extrato concentrado com a matriz encapsulante foi feita com o auxílio de agitador magnético em um recipiente hermeticamente fechado, evitando assim a incorporação de ar na amostra.

\section{Secagem das antocianinas encapsuladas}

O material encapsulado foi seco em "Spray dryer" (Lab Plant SD-0,5). As condições operacionais de secagem foram: temperatura do ar de secagem de entrada $180^{\circ} \mathrm{C}$ e saída $90^{\circ} \mathrm{C}$; pressão de atomização: 0.08 a 0.14 bar; vazão média do ar de secagem: $75.031 \mathrm{~m}^{3} / \mathrm{h}$; vazão média de alimentação: $0.08 \mathrm{~L} / \mathrm{h}$.

\section{Determinações físico-químicas dos pigmentos desidratados}

Para caracterizar o pigmento seco efetuaram-se as seguintes determinações: umidade (BRASIL, 2005); atividade de água: segundo procedimento do aparelho
Aqua Lab Cx-2 Water Activity - System, calibração com solução de $\mathrm{K}_{2} \mathrm{CO}_{3} \cdot 2 \mathrm{H}_{2} \mathrm{O}(420 \mathrm{~g}$ em $100 \mathrm{~mL}$ de água $)$ e leitura da $\mathrm{Aw} / \mathrm{T}\left({ }^{\circ} \mathrm{C}\right)$ das amostras; estabilidade de antocianinas através do teor antocianinas totais e determinação dos índices de cor (colorímetro Chroma Meter CR-400): L* (brancura e/ou luminosidade); a* (a+:cromaticidade predominante para o vermelho, a-: cromaticidade predominante para o verde) e o $b^{*}(b+$ :cromaticidade predominante para o amarelo, $\mathrm{b}$ - : cromaticidade predominante para o azul).

\section{RESULTADOS E DISCUSSÃO}

Apresenta-se na Tabela 1 o planejamento experimental do tipo Taguchi, realizado com as respectivas respostas em concentração de antocianinas, onde a concentração máxima de antocianinas totais obtidas foi de $300 \mathrm{mg} / 100 \mathrm{~g}$ nos níveis inferiores de $\mathrm{pH}(1,0)$ e tempo $(3$ horas) e nos níveis superiores de volume de etanol (250 $\mathrm{mL})$ e temperatura $\left(35^{\circ} \mathrm{C}\right)$, a qual corresponde ao ensaio 7. Muñoz-Espada et al. (2004) encontraram valores próximos ao do presente estudo, utilizando casca (bagaço) da uva cultivar "Concord", onde obtiveram teor de antocianinas totais de $330 \mathrm{mg} / 100 \mathrm{~g}$. A literatura relata que a quantidade e a composição das antocianinas presentes nas uvas e/ou bagaço diferem de acordo com a espécie, cultivar, maturidade, condições climáticas (MATTIVI et al., 2006; MAZZA, 1995; MUÑOZ-ESPADA et al., 2004; RIZZON et al., 1998). O conteúdo de antocianinas, em uvas tintas, varia de 30 a $750 \mathrm{mg}$ por $100 \mathrm{~g}$ da fruta madura. Em uvas "Concord", os valores variaram entre 61 a $112 \mathrm{mg} / 100 \mathrm{~g}$,

Tabela 1 - Planejamento experimental com as respectivas respostas em antocianinas totais.

\begin{tabular}{|c|c|c|c|c|c|}
\hline \multirow[t]{2}{*}{ Ensaio } & \multicolumn{4}{|c|}{ Variáveis independentes } & \multirow{2}{*}{$\begin{array}{c}\text { Resposta } \\
\text { Antocianinas } \\
\text { totais }(\mathrm{mg} / 100 \mathrm{~g})\end{array}$} \\
\hline & $\mathrm{X}_{1}$ & $\mathrm{X}_{2}$ & $\mathrm{X}_{3}$ & $\mathrm{X}_{4}$ & \\
\hline 1 & $2,0(+1)$ & $100(-1)$ & $3(-1)$ & $15(-1)$ & 101 \\
\hline 2 & $2,0(+1)$ & $100(-1)$ & $7(+1)$ & $35(+1)$ & 183 \\
\hline 3 & $2,0(+1)$ & $250(+1)$ & $3(-1)$ & $35(+1)$ & 117 \\
\hline 4 & $1,0(-1)$ & $250(+1)$ & $7(+1)$ & $15(-1)$ & 117 \\
\hline 5 & $1,0(-1)$ & $100(-1)$ & $3(-1)$ & $35(+1)$ & 210 \\
\hline 6 & $1,0(-1)$ & $100(-1)$ & $7(+1)$ & $15(-1)$ & 274 \\
\hline 7 & $1,0(-1)$ & $250(+1)$ & $3(-1)$ & $15(-1)$ & 300 \\
\hline 8 & $1,0(-1)$ & $250(+1)$ & $7(+1)$ & $35(+1)$ & 257 \\
\hline 9 & $1,5(0)$ & $175(0)$ & $5(0)$ & $25(0)$ & 197 \\
\hline 10 & $1,5(0)$ & $175(0)$ & $5(0)$ & $25(0)$ & 223 \\
\hline 11 & $1,5(0)$ & $175(0)$ & $5(0)$ & $25(0)$ & 185 \\
\hline
\end{tabular}

$\mathrm{X}_{1}=\mathrm{pH} ; \mathrm{X}_{2}=$ volume de etanol $(\mathrm{mL}) ; \mathrm{X}_{3}=$ tempo $(\mathrm{h}) ; \mathrm{X}_{4}=$ temperatura $\left({ }^{\circ} \mathrm{C}\right)$. 
enquanto que, em uvas viníferas como "Pinot Noir", "Cabernet Sauvignon" e "Vincent", apresentaram-se concentrações médias de antocianinas de 33, 92 e 439 mg/ $100 \mathrm{~g}$, respectivamente (MAZZA, 1995). Recentemente, Mattivi et al. (2006) encontraram para uvas viníferas "Cabernet Sauvignon", "Syrah", "Cabernet Franc", "Merlot" e "Pinot Noir" médias de antocianinas de 215, 234, 179, 115 e $99 \mathrm{mg} / 100 \mathrm{~g}$, respectivamente.

Após análise estatística, verificou-se que o $\mathrm{pH}$ foi a única variável independente estatisticamente significativa no processo de extração. Seu efeito foi negativo significativo $(\mathrm{p}<0,05)$, indicando que um deslocamento do nível de $\mathrm{pH}$ estudado para valores inferiores, resultaria em um aumento da concentração de antocianinas totais. Como o nível inferior é 1,0, esse foi fixado no menor valor, pois valores inferiores a esse podem resultar, segundo Revilla et al. (1998), em hidrólise parcial das antocianinas aciladas e, conseqüentemente, na estimação incorreta do total de antocianinas. O mesmo autor cita ainda que as antocianinas, em pH baixo, encontram-se, predominantemente, na forma de cátion flavílio, que apresenta coloração vermelha em solução aquosa. O fato de o cátion flavílio ser estável em pH ácido levou à utilização de solventes contendo ácidos orgânicos ou minerais, na extração das antocianinas de frutas e vegetais (MACHEIX et al., 1990), sendo que o uso de solventes contendo ácido fórmico ou clorídrico tem sido indicado para a extração de antocianinas de uvas (REVILLA et al., 1998). Segundo Araújo (1995) a coloração vermelha das antocianinas é perdida em $\mathrm{pH}$ superior a 4,0, para aplicação geral o pH entre 1,0 e 3,0 confere melhor estabilidade às antocianinas. Em pH alto, esse cátion é convertido em outras espécies incolores (REVILLA et al., 1998).
Como a temperatura de extração não influenciou significativamente $(\mathrm{p}<0,05)$, fixou-se a temperatura em $25^{\circ} \mathrm{C}$, por ser a mais fácil de ser obtida experimentalmente. $\mathrm{O}$ mesmo acontecendo com o tempo, que após a análise foi fixado em 3 horas. Em relação ao volume de etanol, esse foi fixado em $100 \mathrm{~mL}$ por resultar em teores de antocianinas obtidos relevantes; e como esse não é uma variável estatisticamente significativa no processo, é interessante economicamente utilizar volumes menores do mesmo.

\section{Encapsulamento e secagem do pigmento}

Observa-se que o ensaio 4 (Tabela 2) resultou em uma maior concentração de antocianinas totais após secagem do pigmento, no qual atingiu-se um valor de $160 \mathrm{mg} / 100 \mathrm{~g}$, para as condições de encapsulamento, utilizando-se somente maltodextrina e $70 \mathrm{~mL}$ de extrato previamente extraído. Observou-se também que, quando somente a goma foi utilizada no encapsulamento, os resultados obtidos em relação às antocianinas totais foram os menores (ensaios 1 e 3 ).

Nos ensaios 2 e 4, observou-se aderência do pó encapsulado nas paredes da câmera de secagem e conseqüentemente perdas do material encapsulado, isso sendo mais pronunciado no ensaio 4. Essa aderência, possivelmente, esteja associada ao elevado teor de volume de extrato $(70 \%, v / v)$, que apresenta, em sua composição, açúcares (frutose e glicose), consequentemente ocasionando caramelização dos mesmos. A aderência observada também poderá estar relacionada à mudança de estado físico, pois segundo Magalhães Netto (1997), estruturas amorfas ou parcialmente amorfas são formadas em vários processos como a desidratação (Spray-dryer). A remoção da água durante a manufatura produz esse tipo de estrutura. A temperatura onde acontece essa mudança

Tabela 2 - Planejamento experimental fatorial com a resposta em antocianinas totais.

\begin{tabular}{cccc}
\hline Ensaios & \multicolumn{2}{c}{ Variáveis independentes* } & Resposta \\
\cline { 2 - 3 } & $\mathrm{X}_{5}$ & $\mathrm{X}_{6}$ & $\begin{array}{c}\text { Antocianinas totais } \\
(\mathrm{mg} / 100 \mathrm{~g})\end{array}$ \\
\hline 1 & $0: 100(-1)$ & $30(-1)$ & $78,7 \pm 0,81$ \\
2 & $100: 0(+1)$ & $30(-1)$ & $87,1 \pm 0,14$ \\
3 & $0: 100(-1)$ & $70(+1)$ & $44,6 \pm 1,9$ \\
4 & $100: 0(+1)$ & $70(+1)$ & $159,9 \pm 1,9$ \\
5 & $50: 50(0)$ & $50(0)$ & $89,0 \pm 0,67$ \\
6 & $50: 50(0)$ & $50(0)$ & $103,1 \pm 3,6$ \\
7 & $50: 50(0)$ & $50(0)$ & $101,6 \pm 2,8$ \\
\hline
\end{tabular}

\footnotetext{
$* \mathrm{X}_{5}=$ maltodextrina:goma; $\mathrm{X}_{6}=$ volume de extrato $(\mathrm{mL})$.
} 
de estado físico é chamada de temperatura de transição vítrea $\left(\mathrm{T}_{\mathrm{g}}\right)$. Essas alterações incluem mudanças nas propriedades mecânicas como colapso, aglomeração, resultantes das modificações nas estruturas ou fluxo viscoso. Mudanças na difusão causam cristalização de açúcares amorfos e, possivelmente, modificações na cinética de algumas reações.

O mesmo autor afirma que a presença de carboidratos de peso molecular mais alto, como a maltodextrina, gomas e outros materiais encapsulantes, contribuem para auxiliar na estabilidade do sistema aumentando sua $\mathrm{T}_{\mathrm{g}}$. Porém neste estudo, os melhores resultados foram observados quando utilizou-se uma porcentagem de goma arábica associada à maltodextrina, referente ao ponto central do planejamento de experimentos (ensaios 5, 6 e 7), o que propiciou uma menor perda de material por aderência.

Os dados foram analisados estatisticamente, onde verificou-se que o volume de extrato não se apresentou significativo $(\mathrm{p}<0,05)$ no teor de antocianinas totais encapsulada dentro da faixa estudada, logo esse fator foi retirado da regressão e adicionado à falta de ajuste. A Equação 2 apresenta o modelo codificado não linear para o teor de antocianinas totais, em função dos fatores estudados, no qual verifica-se que o efeito de interação significativo entre o volume de extrato e relação maltodextrina:goma.

$\mathrm{AT}=94,9+30,9 \cdot \mathrm{X}_{5}+26,7 . \mathrm{X}_{5} \cdot \mathrm{X}_{6}$

Onde: AT $=$ Antocianinas totais $(\mathrm{mg} / 100 \mathrm{~g}) ; \mathrm{X}_{5}=$ concentração de maltodextrina:goma; $\mathrm{X}_{6}=$ volume de extrato $(\mathrm{mL})$.

O modelo codificado apresentado na Equação 2 foi validado $(\mathrm{p}<0,05)$ pela análise de variância, na qual verificou-se que o F calculado apresentou-se 3,5 vezes superior ao $\mathrm{F}$ tabelado e o coeficiente de correlação obtido foi de 0,92 . Observa-se uma tendência de aumento no teor de antocianinas totais quando o volume de extrato inicialmente utilizado é maior. Observa-se também que a utilização de apenas maltodextrina no encapsulamento resulta em uma maior eficiência do mesmo, sendo que a combinação dela com a goma gera teores intermediários de antocianinas totais e a utilização da goma somente acarreta uma diminuição no teor de antocianinas final. Porém, conforme discutido anteriormente, experimentos somente com maltodextrina ocasionaram perdas de material na câmara de secagem do Spray-dryer e conseqüente escurecimento do pigmento por caramelização dos açúcares.

Em todos os experimentos referentes ao planejamento de experimentos foram também avaliados o teor de umidade e a atividade de água, sendo que esses foram inferiores a $5,0 \%$ e 0,266 , respectivamente.

Observam-se na Tabela 3 as demais variáveis dependentes que foram analisadas de acordo com o planejamento experimental realizado.

Em relação ao índice $\mathrm{L}^{*}$, que mede a brancura e/ou luminosidade, verificou-se que o menor valor obtido foi no ensaio 4, ensaio este correspondente ao maior teor de antocianinas totais obtidas, porém com aspecto visual de coloração mais escura. Os valores obtidos para essa resposta foram tratados estatisticamente, sendo que apresenta-se na Equação 3 o modelo codificado obtido para a índice $\mathrm{L}^{*}$ em relação às variáveis estudadas, onde nota-se que os efeitos principais de ambas as variáveis estudadas apresentam-se significativos $(\mathrm{p}<0,05)$, bem como o efeito de interação entre as variáveis. O modelo obtido foi validado pela análise de variância, que apresentou um coeficiente de correlação de 0,98 , onde verifica-se que

Tabela 3 - Planejamento experimental fatorial completo e respostas em índices de cor.

\begin{tabular}{|c|c|c|c|c|c|}
\hline \multirow[t]{3}{*}{ Ensaios } & \multicolumn{2}{|c|}{ Variáveis independentes } & \multicolumn{3}{|c|}{ Respostas } \\
\hline & & & \multicolumn{3}{|c|}{ Índice de cor } \\
\hline & & & $\mathrm{L}^{*}$ & $a^{*}$ & $\mathrm{~b}^{*}$ \\
\hline 1 & $0: 100(-1)$ & $30(-1)$ & 73,7 & 17,2 & 4,1 \\
\hline 2 & $100: 0(+1)$ & $30(-1)$ & 71,4 & 13,1 & 3,6 \\
\hline 3 & $0: 100(-1)$ & $70(+1)$ & 63,1 & 37,8 & 4,2 \\
\hline 4 & $100: 0(+1)$ & $70(+1)$ & 49,4 & 31,7 & 1,3 \\
\hline 5 & $50: 50(0)$ & $50(0)$ & 61,4 & 29,4 & 1,6 \\
\hline 6 & $50: 50(0)$ & $50(0)$ & 61,9 & 30,2 & 0,44 \\
\hline 7 & $50: 50(0)$ & $50(0)$ & 63,2 & 28,1 & 0,62 \\
\hline
\end{tabular}

\footnotetext{
$* \mathrm{X}_{5}=$ maltodextrina:goma; $\mathrm{X}_{6}=$ volume de extrato $(\mathrm{mL})$.
} 
o valor de F calculado foi cerca de 3,7 vezes superior ao valor tabelado.

$$
L^{*}=63,4-3,9 . X_{5}-8,2 \cdot X_{6}-2,8 . X_{5} . X_{6}
$$

Onde: $\mathrm{L}^{*}=$ índice de cor (brilho e/ou luminosidade); $\mathrm{X}_{5}=$ relação maltodextrina:goma; $\mathrm{X}_{6}=$ volume de extrato $(\mathrm{mL})$.

Observa-se na Equação 4 o modelo codificado que descreve o comportamento do índice de cor a* em relação as variáveis relação maltodextrina:goma e volume de extrato dentro da faixa estudada. O modelo obtido foi validado pela análise de variância, que apresentou um coeficiente de correlação de 0,96 , onde verifica-se que o valor de $\mathrm{F}$ calculado foi cerca de 3,4 vezes superior ao valor tabelado. Observa-se que à medida que se aumenta o volume de extrato, maior o índice de cor $\mathrm{a}^{*}$. Têm-se também faixas aceitáveis desse índice quando são utilizadas proporções iguais de maltodextrina e goma.

$a^{*}=26,8-2,6 \cdot X_{5}+9,8 . X_{6}$

Onde: $\mathrm{a}^{*}=$ índice de cor (predominante vermelho); $\mathrm{X}_{5}=$ relação maltodextrina:goma; $\mathrm{X}_{6}=$ volume de extrato $(\mathrm{mL})$.

\section{CONCLUSÕES}

A concentração máxima de antocianinas totais obtidas foi de $300 \mathrm{mg} / 100 \mathrm{~g}$ de bagaço de uva (umidade $5 \%$ ), nas condições de $\mathrm{pH}$ 1,0, tempo de extração de 3 horas, temperatura de $15^{\circ} \mathrm{C}$ e volume de etanol de $250 \mathrm{~mL}$.

A melhor condição para o encapsulamento e a secagem foi quando utilizaram-se proporções iguais de maltodextrina e goma arábica na qual o encapsulado apresentou $95 \mathrm{mg}$ de antocianinas $/ 100 \mathrm{~g}$.

\section{REFERÊNCIAS BIBLIOGRÁFICAS}

ARAÚJO, J. M. A. Química de alimentos: teoria e prática. Viçosa: UFV, 1995. 365 p.

BARBOSA, M. I. M. J.; BORSARELLI, C. D.; MERCADANTE, A. Z. Light stability of spray-dried bixin encapsulated with different edible polysaccharide preparations. Food Research International, Madison, v. 38, p. 989-994, 2005.

BOBBIO, F. O.; BOBBIO, P. A. Química do processamento de alimentos: pigmentos. São Paulo: Varela, 2001.

BRASIL. Ministério da Saúde. Agência Nacional de Vigilância Sanitária. Métodos físico-químicos para análise de alimentos. 4. ed. Brasília, DF, 2005.
CARAMEZ, R. R. B. Caracterização físico-química e estudo da estabilidade das antocianinas do cálice de Hibiscus sabdariffa L. 1999. 101 f. Dissertação (Mestrado em Ciência de Alimentos) - Universidade Federal de Santa Catarina, Florianópolis, 1999.

COUTINHO, M. R. Obtenção de antocianinas presentes no repolho roxo. 2002. 80 f. Dissertação (Mestrado em Engenharia de Alimentos) - Universidade Federal de Santa Catarina, Florianópolis, 2002.

DEL-VALLE, E. M. M. Cyclodextrins and their uses: a review. Process Biochemistry, Amsterdam, v. 39, p. 10331046, 2004.

FALCÃO, L. D. et al. Copigmentação intra e intermolecular de antocianinas: uma revisão. Boletim do CEPPA, [S.1.], v. 21, n. 2, p. 351-366, 2003.

GAO, L.; MAZZA, G. Extraction of anthocyanin pigments from purple sunflower hulls. Journal of Food Science, London, v. 61, p. 600-603, 1996.

GUIMARÃES, I. S. S.; CARVALHO, M. P. M.; SUNDFELD, E.; PHILIP, T. Corantes naturais para alimentos: antocianinas de cascas de uvas. Boletim de Pesquisa da Embrapa, Rio de Janeiro, n. 11, p. 29, 1984.

HARBORNE, J. B. The flavonoids: recent advances. Plant Pigments, London, p. 298-343, 1988.

HUMEAUT, C.; ROVEL, B.; GIRARDIN, M. Enzimatic esterification of bixin by 1-ascorbic acid. Biotechnology Letters, Easton, v. 22, n. 2, p. 165-168, 2000.

LOPES, R. M.; OLIVEIRA, T. T.; NAGEM, T. J.; PINTO, A. S. Flavonóides. Biotecnologia, Ciência \& Desenvolvimento, [S.1.], v. 3, n. 14, p. 18-22, 2000.

MACHEIX, J. J.; FLEURIET, A.; BILLOT, J. Fruit phenolics. Boca Raton: CRC, 1990. 378p.

MAGALHÃES NETTO, F. Influência da atividade de água na temperatura vítrea. In: JARDIM, D. C. P.; GERMER, S. P. M. Atividade de água em alimentos. Campinas: ITAL, 1997. p. 4-14.

MALACRIDA, C. R.; MOTTA, S. da. Antocianinas em suco de uva: composição e estabilidade. Boletim do CEPPA, [S.1.], v. 24, n. 1, p. 59-82, 2006. 
MANTELL, C.; RODRIGUEZ, M.; MARTINEZ DE LA OSSA, E. Semi-batch extraction of anthocyanins from red grape pomace in packed beds: experimental results and process modeling. Chemical Engineering Science, [S.1.], v. 57, p. 3831-3838, 2002.

MATTIVI, F.; GUZZON, R.; VRHOVSEK, U.; SEFANINI, M.; VELASCO, R. Metabolite profiling of grape: flavonols and anthocyanins. Journal of Agriculture and Food Chemistry, [S.1.], 2006. In press.

MAZZA, G. Anthocyanins in grapes and grape products. Critical Review of Food Science and Nutrition, Madison, v. 35, p. 341-371, 1995.

MUÑOZ-ESPADA, A. C.; WOOD, K. V.; BORDELON, B.; WATKINS, B. A. Anthocyanin quantification and radical scavenging capacity of Concord, Norton, and Marechal Foch grapes and wines. Journal of Agriculture and Food Chemistry, Easton, v. 52, n. 22, p. 6779-6786, 2004.

NIKETIC-ALEKSIC, G.; HRALDINA, G. Quantitative analysis of the antocyanin content in grape juices and wines. Lebensm. Wiss. U.Technol., [S.1.], v. 5, p. 163-165, 1972.
O’BOYLE, A. R.; KASSAM, N. A.; RUBIN, L. J.; DIOSADY, L. L. Encapsulated cured-meatpigment and its application in nitrite-free ham. Journal of Food Science, London, v. 57, n. 4, p. 807-812, 1992.

REVILLA, E.; RYAN, J. M.; MARTIN-ORTEGA, G. Comparison of several procedures used for the extraction of anthocyanins from red grape. Journal of Agriculture and Food Chemistry, Easton, v. 46, p. 45924597, 1998.

RIZZON, L. A.; MANFROI, V.; MENEGUZZO, J. Elaboração de suco de uva na propriedade veicula. Bento Gonçalves: Embrapa Uva e Vinho, 1998. 24 p.

WAGNER, L. A.; WATHESEN, J. J. Stability of spray-dried encapsulated carrot carotene. Journal of Food Science, London, v. 60, n. 5, p. 1048-1053, 1995.

XAVIER, F. M. Estudo da extração de antocianinas em colunas recheadas. 2004. 135 f. Dissertação (Mestrado em Engenharia Química) - Universidade Federal de Santa Catarina, Florianópolis, 2004. 